The Council has approved the election of the following candidates to the grade of Member.

Avery, Brian A.

Baker, John M.

Balon, Michael J.

Barr, Alan G.

Baruffaldi, Robert M.

Benca, Robert A.

Bennett, Judy L.

Bitter, Kimball B.

Bowers, Gary W.

Bowley, Clinton J.

Brown, Ellen B.

Burzinski, Mark J.

Cai, Xiaming

Carbin, Gregory W.

Carlson, David J.

Carver, Donald R.

Casey, Leah W.

Cheng, Xinhua

Christensen, Eric J.

Clark, Kenneth M.

Clary, Richard D.

Cohen, Robert Alan

Cohn, Stephen Arthur

Collins, Michael P.

Cornick, Jon C.

Craft, David L.

Cramer, James $\mathrm{H}$.

Cullather, Richard I.

DeWald, Van L.

Dean, Devin B.

Dennis, Harrison J.

DiNardo, Matthew S.

Ding, Hanjun

Donahoe, Christopher S.

Dubberke, Donna M.

Duthie, James $M$.

Ecklund, Warner L.

Fahlbusch, Frederick L.

Ferrara, William J.

Flack, Catherine A.

Foley, Jonathan A.

Foster, Matthew W.

Freeman, David W.

Gaffin, David M.

Galindo, Ignacio

Gallegos, Rafael L.

George, Jr., David L.

Georgiadis, Teodoro

Goehrs, Liesa R.
Golnaraghi, Maryam

Graham, Randy A.

Griffies, Stephen M.

Griffith-Tesch, Heather E.

Hamann, Donald J.

Hastings, John P.

Helfrich, Sean R.

Hewitt, Joseph A.

Hubright, Jeffrey L.

Innocentini, Valdir

Ingram, John J.

Iskra, Mark A.

Istok, Michael J.

Kaster, Mark A.

Kattawar, Paul F.

Keen, Ann Barbara

Keislar, Robert Evan

Kelly, Kathryn A.

Kim, Joon

Kimbrough, Jr., William F.

Kinsella, Ingrid A.

Klasson, Michael D.

Koch, Rob E.

Kopecek, Joseph J.

Kosek, James P.

Kousky, Jamie V.

Krafton, William J.

Kramer, Marc S.

Kroll, James T.

Kucera, Paul A.

Kuhn, Ronald E.

Kuji, Makoto

Lam, Shu Hong

Lean, Judith L.

Lee, Hai-Tien

Lee, Jin-Luen

Leone, Michael Guerino

Lewis, Peter J.

$\mathrm{Li}$, Xiaofan

Lim, Hyo-Suk

Lin, Ho

Livesay, Jess $\mathrm{H}$.

Loehrer, Scot M.

Lund, Donald E.

Luo, Gang

Lynch, Marcus W.

Lynn, Kris

Lyons, Jeffrey P.

Martin, Derrel R.
Marz, Loren Carl

McKay, Megan

Mearns, Linda $\mathrm{O}$.

Meys, Anthony W.

Miller, Jerral $\mathrm{J}$.

Miller, Rosemary A.

Milman, Andrew S.

Moise, Vincent $F$.

Moritz, Gwen M.

Murray, William E.

Musembi, David K.

Musick, David R.

Nespor, Jerald D.

Nicholls, Joe R.

Noh, Yign

O'Lenick, Michael P.

Ohandley, Chris

Oki, Taikan

Oldshue, John $\mathrm{H}$.

Onek, Austen L.

Osterhouse, Michael P.

Otto, Paul A.

Ovens, David W.

Payne, Robert A.

Pelatti, Rocco D.

Pfeifer, Gail M.

Powell, Patrick T.

Presnell, David W.

Press, Timothy $F$.

Price, Colin G.

Provis, David G.

Quintana-Gomez, Ramon A.

Rappengluck, Bernhard

Reeder, Andrew E.

Rinaldi, James F.

Ritz, Scott A.

Robeson, Scott M.

Robinson, Ian Stuart

Rudack, David E.

Ryuta, Kurora

Saito, Mark I.

Sauer, Thomas J.

Sautter, David C.

Sawyer, Jonathan L.

Schrumpf, Bradford D.

Schweder, York E.

Seo, Dong-Jun

Shi, Jainn Jong

Shie, Chung-Lin
Shriver, Jay F.

Sica, Robert J.

Sidney, Orelon A.

Skloot, Gerald D.

Sluben, Jr., Richard J.

Smalstig, Jonathan J.

Smith, Peter Daniel

Smith, Timothy W.

Solomon, Joe K.

Song, Ran

Spano, Donatella

Spriggs, Thomas E.

Sprintall, Janet

Stephens, Scott E.

Stoll, Peter J.

Sunwoo, Young

Svercl, Stephen J.

Sweeney, Shannon R.

Tanimoto, Youichi

Tibbetts, Robert Thomas

Trautwein, Kenneth R.

Vaughan, Jr., Leonard C.

Vera, Carolina

Vicente, Manuel de Jesus

Waller, Matthew J.

Walshe, Patrick T.

Wang, Henry Hui-Ju

Weinstein-Lloyd, Judith B.

West, Derek A.

Whelan, Christopher P.

Wicker, John L.

Williams, Albert J.

Williams, Eric J.

Wilson, Lyle D.

Winstead, Curtis M.

Wright, Ryan E.

Wrightson, Robert C.

Wrona, Richard A.

Wu, Xiao Qing

Xie, Lian

Xie, Rongrong

Yang, Xiaohua

Yang, Zong-Liang

Yuan, Yeli

Yun, Jae-Yul

Zahren, David R.

Zhong, Shiyuan

Zhou, Xiaojing

Zitzmann, Richard F. 
The Council has approved the election of the following candidates to the grade of Student Member.

\begin{tabular}{|c|c|c|c|}
\hline Alkhalil, Adoum & Emeagwali, Philip C. & Lawson, Erika D. & Quaintance, George W. \\
\hline Archer, Scott M. & Eyerman, Kathleen L. & Lee, Jae-Won & Rao, Pejaver A. \\
\hline Bantzer, Christian J. & Faber, Terry A. & Lenning, Eric M. & Renwick, James A. \\
\hline Barry, Timothy S. & Fox, Patricia A. & Levine, Steven M. & Riley, Kevan P. \\
\hline Basich, Francis M. & Franzen, Michelle $\mathrm{M}$. & Lewis, Michael M. & Roberts, Jane E. \\
\hline Bator, Aaron & Gamgort, Patrick & Li, Yonghong & Roberts, Leslea A. \\
\hline Baxter, Barry N. & Gaudenzi, Dennis A. & Magsig, Michael A. & Rohli, Robert V. \\
\hline Bennett, Stephen D. & Geiger, III, Walter E. & Manino, Robert V. & Romig, Kevin D. \\
\hline Berardelli, Jeffrey R. & Gibson-Wilde, Dorothy E. & Mathewson, Timothy $\mathrm{O}$. & Rzonca, Marek D. \\
\hline Bettes, Robert L. & Ham, Mallory J. & Matthews, John C. & Sathiyamoorthy, \\
\hline Bicknell, Joel R. & Handley, Lawrence A. & May, Lisa M. & Sudharshan \\
\hline Blaes, Jonathan L. & Haner, Andrew W. & McCarthy, Ann K. & Saulo, Andrea C. \\
\hline Blanchard, David A. & Hanke, Markus H. & McDonnell, David M. & Seifert, Konstanze $\mathrm{H}$. \\
\hline Bogner, Paul B. & Henderson, Patricia K. & Mclnerney, Mark A. & Siffer, Alan M. \\
\hline Bourne, Kevin T. & Henson, Tanja E. & McLaughlin, John B. & Sinnema, Jeremy D. \\
\hline Bramer, Daniel J. & Herndon, John J. & Metzger, Eric L. & Smith, Scott P. \\
\hline Brenner, Joseph R. & Hess, Travis R. & Miller, Ryan L. & Sowers, Michael B. \\
\hline Brooks, Robyn H. & Hodges, Gary B. & Mischna, Michael A. & Starr, Diana L. \\
\hline Brown, David John & Hollinger, Christopher L. & Moats, Kristi A. & Starr, Kristina M. \\
\hline Brown, Mike E. & Hsu, Kuo-lin & Moliterno, Nicholas G. & Stephenson, Gary F. \\
\hline Jonathan L. & $\mathrm{Hu}$, Yuanlong & Moore, Maureen E. & Taylor, Jason D. \\
\hline Cash, Benjamin A. & Huang, Jin & Morawitz, Werner M. & Vaughan, Joseph K. \\
\hline Castaneda, Maria E. & Hughes, Brian $\mathrm{K}$. & Moyer, Benjamin W. & Vizy, Edward K. \\
\hline Chan, William & Hunt, Gregory L. & Mundhenk, Katherine E. & Walden, Von P. \\
\hline Cipriano, Chadwick A. & Hwu, Wenje & Nelson, Heather A. & Walsh, Abby C. \\
\hline Clauss, Brad H. & Jamason, Paul F. & Organista, Salvador & Walters, Tamera A. \\
\hline Clement, Amy C. & Jiang, Weidong & Orrock, Jeffrey A. & Wanamaker, James R. \\
\hline Cooper, Steven J. & Jin, Yao & Overstreet, John C. & Wang, Houjun \\
\hline Csurics, David A. & Johnson, Rodney J. & Pan, Zai Tao & Wang, Junhong \\
\hline Deal, III, Roy L. & Kaplan, Daniel A. & Pawlak, Daniel T. & White, Kate \\
\hline DelSoldato-Knopf, Sherri A. & Kappel, Bill D. & Pawloski IV, Adam S. & Xu, Fan \\
\hline Delfelder, Steven M. & Kellerhols, Morkus O.B. & Paxson, Jason D. & Yeh, Hsi-Chyi \\
\hline Dipasquale, Neal E. & Kilby, James R. & Perfater, Sarah E. & Zaff, David A. \\
\hline Downs, William N. & Kim, Seong-Joong & Peterson, Barry T. & Zahariev, Konstantin \\
\hline Drury, Systke & Kimberlain, Todd B. & Pittalwala, Iqbal I. & Zhu, Tong \\
\hline Eastin, Matthew D. & Kubat, Gary B. & Porter, Christopher W. & Zidle, Matthew L. \\
\hline
\end{tabular}

The Council has approved the election of the following candidates to the grade of Member with Student Privileges.

$\begin{array}{llll}\text { Amitai, Eyal } & \text { Randell, Scot C. } & \text { Segal, Yaron } & \text { Wikle, Christopher K. } \\ \text { Ding, Wen } & \text { Schmitz, Jeffrey T. } & \text { Skarvelis, loannis } & \text { Yoshitsugu, Ellen }\end{array}$

Huang, Ho-Chun

The Council has approved the election of the following candidates to the grade of Corporation Member. 
The Council has approved the election of the following candidates to the grade of Associate Member.

\author{
Aktary, Nacim \\ Blake, Eric S. \\ Bourgeous, Brent D. \\ Bovitz, Christopher T. \\ Brescia, Michael A. \\ Broyles, John C. \\ Burgett, Wesley S. \\ Cantrell, Jr., Louis E. \\ Capotondi, Antonietta \\ Cempa, Michael J. \\ Cherington, Michael \\ Chester, David Brian \\ Chilson, Phillip B. \\ Choi, Jun-Tae \\ Christopher, Sundar A. \\ Crum, Jeffrey B. \\ DeBroder, Charles E. \\ Eltahir, Elfatih Ali Babiker
}

\author{
Moore, Cam H. \\ Moran, Marc G. \\ Murphy, Mike W. \\ Myers, Timothy L. \\ Nagler, Lewis $\mathrm{H}$. \\ Nicco, Mike T. \\ Niemira, Michael $P$. \\ Nocera, Frank M. \\ O'Shay, Adam J. \\ Oberson, Fredric M. \\ Pickens, Wendell K. \\ Proud, Michael J. \\ Reider, Adam J. \\ Reimer, Gilles P. \\ Richardson, James $L$ \\ Riley, David J. \\ Roer, Jason C.
}

\author{
Roth, Sandra I. \\ Sargis, Jr., Robert S. \\ Smith, Robert M. \\ Solomon, Samuel A. \\ Sostre, Sol L. \\ Stavish, Michael T. \\ Steele, Scott J. \\ Talone, Gregory J. \\ Tarbell, Susan A. \\ Throop, Michael E. \\ Valle, Christopher \\ Wolfe, Bill W. \\ $\mathrm{Wu}$, Xiaohua \\ Yu, Jia-yuh \\ Yu, Jin-Yi \\ Zhang, Renyi \\ Zoltowski, Jeffrey A.
}

\section{EMPLOYMENT ANNOUNCEMENTS}

\section{The American Meteorological Society publishes a monthly newsletter announcing employment opportunities for positions in the atmospheric and oceanic fields.}

Members and nonmembers may subscribe to the announcements at an annual (12 monthly issues) subscription rate of $\$ 15$ for AMS members and $\$ 30$ for nonmembers (Both members and nonmembers, kindly add $\$ 10$ for airmailing to a foreign address). After July 1, half-year subscriptions (for July through December issues) are available at one half of the above annual prices

Organizations with openings to be filled by qualified individuals seeking positions in the atmospheric and related fields should contact the American Meteorological Society, 45 Beacon Street, Boston, MA 02108-3693, 617-2272426, ext. 250 for announcement guidelines and charges. ASCII diskettes are invited. You may also submit announcements via internet: amsempl@aip.org. 